\title{
Product development cost estimation and optimisation in a global manufacturing environment
}

\author{
Y.L. Tu and S.Q. Xie \\ Department of Mechanical Engineering, University of Canterbury, Private Bag 4800, \\ Christchurch, New Zealand \\ Email: p.tu@mech.canterbury.ac.nz.
}

\begin{abstract}
In the early stages of a product development process, the estimate of a developing product life cycle cost is the primary information which will be needed to determine the profitability of the product. The more reliable is the cost estimate process, the more likely the right decision will be made. This has become an important issue for rapid product development. In this paper, an Internet based cost estimation and optimisation system will be reported, which is a subsystem of the Rapid Product Development system for one-of-a-kind production (OKP) developed at the University of Canterbury. The product development cost under consideration in this paper is the sum of the costs of product design, production and logistics, which is the main cost of a product. Some other possible costs associated with a product development cycle, e.g. overhead costs, are normally proportional to this main product development cost and in practice they are estimated by multiplying a constant percentage to the product development cost. Unlike some earlier research which focused on the cost estimate in a single manufacturing company, this Internet based cost estimation system aims at the product development cost estimate and optimal control under the complexities of a global manufacturing environment. The proposed Cost Estimate and Optimal Control methods and algorithms have been implemented in a sheet metal manufacturing company. The industrial implementation is reported in the end of this paper.
\end{abstract}

\section{Keywords}

Cost estimation and optimisation, one-of-a-kind production (OKP), rapid product development, concurrent engineering, sheet metal manufacturing.

\section{INTRODUCTION}

In today's keen competitive global market, a short product development cycle time and high quality product with a reasonable lower cost are becoming more and more critical for these small and middle sized manufacturing companies to survive. The product development cost normally determines the market share, profit and return on investment of these small and middle-sized manufacturing companies. To control a product development cost at earlier product development stages is in turn a problem on how to accurately estimate a possible cost associated with a product development cycle. Estimate of product development cost is closely related to the 
selection of a mix of production development processes, which can fully meet the customer's requirements and with a lowest sum of the costs of these selected processes. Hence, it is necessary to develop new methods and tools for these small and middle sized manufacturing companies to rationally select proper product development processes, accurately estimate and optimally control the product development cost.

Cost estimation and reduction have been always of great concern in a product development cycle. In recent years, the research which aims to improve a manufacturing company's product development ability has been focused on two aspects, 1) how to shorten the product development cycle time, and 2) how to reduce the product development cost. In fact, these two aspects are interrelated each other. A shorter product cycle time will normally result in a lower product development cost. On the other hand, to optimally control a product development cost usually leads to a shorter product development cycle time. Hence, the research projects, either aim at shortening the product development cycle time or focus on reducing the product development cost, have the same research goal, i.e. rapid and economic product development, just from two different angles.

To estimate and optimally control a product development cost in today's global competitive manufacturing market, several issues need to be researched. First, product development (PD) in a global manufacturing environment has been approved to have the advantages of agility, often providing quick and cheap solutions for some parts or even the whole product development from the partners or sub-contractors, and maximally and yet effectively applying state-of-art technology to address customer's requirements from a global competitive environment. However, a well-established PD management system (e.g. cost estimate and optimal control system) in a global manufacturing environment is still under the development. Although a relative large number of papers and reports were found to address the cost estimate and control problems in various $\mathrm{PD}$ processes, such as design, manufacturing and logistics management, these methodologies were normally developed based on the cases in individual companies rather than in a global manufacturing environment. In a global manufacturing environment, a manufacturing company (or normally called a master company) may carry out a PD cycle through collaboration among its partners or sub-contractors. These partners and sub-contractors may use different PD management systems and computer aided engineering software systems. To effectively communicate, share the necessary information, integrate the efforts and optimally control the PD cost between the master company and its partners/sub-contractors are the problems need to be addressed by this research which aims to develop a PD cost estimate and optimal control system in a global manufacturing environment.

Second, no matter in a global manufacturing environment or in an individual manufacturing company, PD cost estimate and control is an interdependent and correlated problem. It is influenced and dynamically determined by a number of preconditions. The cost of a product design, for instance, will be strongly influenced by the quality of the product definition. A correctly understanding customer's requirements and hence a clear product definition will normally result in a quick and successful product design with a lower product design cost which is mainly determined by the total used engineers' hours (Tu et al. 2002). Likewise, the cost of 
manufacturing a part is normally affected by the design of the part. The final form of the part design, which includes the geometry, surface, tolerance and property specifications of the part, either directly or indirectly influences the selection of manufacturing processes and hence the cost of manufacturing the part. The bad experiences gained from manufacturing industries, particularly from sheet metal manufacturing companies, show that decisions made at the design stage without PD costing considerations normally result in a wrong product development. A wrong PD cycle will let the company suffer a longer PD cycle time due to a lot of reworks, a poorer product design and manufacture which is often indicated by a lower customer's satisfaction, and hence a higher PD cost.

The PD cost estimate and control is no doubt an effective tool to monitor a PD cycle. The cost estimate results can be used as important reference parameters or indicators to check the feasibility and profitability of a developing/new product. It is well experienced that reworks in down stream of a PD process, e.g. reworks in manufacturing, are more costly than correction made in earlier PD stages, e.g. in design. PD cost estimate and control is obvious a planning and control activity which is taken place at earlier PD stages. Hence to accurately estimate and optimally control the cost of a product development is crucial to shorten a PD cycle time and reduce the $\mathrm{PD}$ cost. To develop the useful methods, algorithm and system framework to support this planning and control activity at earlier PD stages is very important for manufacturing companies to improve their market place, particularly for those small and middle sized manufacturing companies.

\section{SYSTEMATIC OVERVIEW}

The cost estimate and optimisation system to be presented in this paper is a part of our overall rapid and economic product development system which was developed mainly for the small and middle sized manufacturing companies to quickly response and meet the customer's needs. The system includes 5 major subsystems, i.e. Product Definition and Design, Manufacturing Process Planning and Optimisation, Operations Management, Cost Estimate and Optimal Control, and Logistics Management. These 5 sub-systems can communicate each other via computer intra/internets. To integrate the information and data flow among these sub-systems, an integrated product data model was developed and presented (Tu 1997b, 1997c, Tu et al. 2000a, 2000b and 2000c). This data model is named as Product Production Structure, which can store all the necessary data through a product life cycle hierarchically in a common object. In this common object, the data are recorded in EXPRESS of STEP (ISO 1994 and 1994E). Hence, all kinds of computer-aided engineering and management software packages should be able to operate this common object. This data model provides feasible solutions to build automatic data links among product design, process planning, logistics management (i.e. supply, partner and sub-contractor chains management), production scheduling and costing.

This cost estimate and optimal control to be presented in this paper will be taken place in earlier PD stages. In these stages, a product is only designed and planned and it will not be actually made. The product design will include the activities like 
product definition, engineering drawings, and specifications of manufacturing and technical requirements. The plan includes process planning, cost estimate and optimisation, shop floor scheduling, inventory check, logistics planning and management. Some shop floor tests may be done to approve some design ideas and manufacturing processes during these earlier stages. A product will be concurrently designed and planned according to a concept called "Prototype based incremental product development". By this concept, a produce will be designed and planned feature by feature. The product design will start with a prototype or several prototypes which are the products made in the past and are similar to the new product. The design process is in fact a modification of the prototypes. Once a feature is modified or a new feature is added by the product design, it also needs to be planned by carrying out all the possible planning activities as mentioned before. This incremental or concurrent way of approaching a product development is proposed to handling the high customisation and great uncertainties in OKP. An OKP company is understood by us as the one who takes product oriented position strategy and engineer to order market strategy. The sheet metal part as discussed later in this paper is a part of a customised or OKP sheet metal product.

It is obvious that the Cost Estimate and Optimal Control system is subsystem of our OKP product development system. Before we discuss this subsystem, we would like to define a PD cost in the following:

$$
\begin{aligned}
& \text { PD cost }=\mathrm{C}_{\mathrm{dd}}+\mathrm{C}_{\mathrm{p}} \\
& \text { Where: } \\
& \mathrm{C}_{\mathrm{dd}}: \text { Product Definition and Design cost. } \\
& \mathrm{C}_{\mathrm{p}} \text { : Product Production cost. }
\end{aligned}
$$

Product definition and design cost is determined by the consumed times of the product design engineers. To directly control these times does not make any sense since the main goal of product definition and design to design a product with a high quality. A high quality product means the product will provide the maximum satisfaction of the customer. Hence we developed a linear programming model to maximize the customer's satisfaction under the constraints of customer's requirements, technical constraints, resource constraints and a given product definition and design budget (measured in dollars) which is normally decided by the company's management according to the company's marketing strategy and past experience (Tu et al. 2002). This means the product definition and design cost will not be estimated and controlled by the Cost Estimate and Optimal Control System as shown in Figure 1. The costing sub-system in the product development system as shown in Figure 1 estimates and controls the total product manufactures and logistics cost.

The product production cost is the sum of the costs of all the manufacturing processes. The cost of a manufacturing process includes:

1. Test and possible rework fees due to the difficulty of the process.

2. The total consumption of the necessary manufacturing resources to carry out the process. It includes the consumptions of workforce, machines, and tools.

3. The total Logistics cost for purchasing and/or transporting a raw-material, component or outsourced manufacturing process, and relevant logistics 
management cost (e.g. communication cost and paper/data management cost) If a manufacturing process is a outsourced or sub-contracted, it only includes this logistics cost. If a raw-material or component is within the company, the logistics cost for this raw-material or component equals to the price of the material or component plus inventory and material handling cost in the company. In short, to simplify the cost estimate and control in a global environment, we consider material handling and purchasing cost as a part of the logistics cost.

\section{COST INDEX STRUCTURE}

To estimate and control the manufacture cost and logistics cost of a product development, a Cost Index Structure was developed and illustrated in Figure 1. In accordance with the Product Production Structure, the Cost Index Structure adopts the same hierarchy of the Product Production Structure.

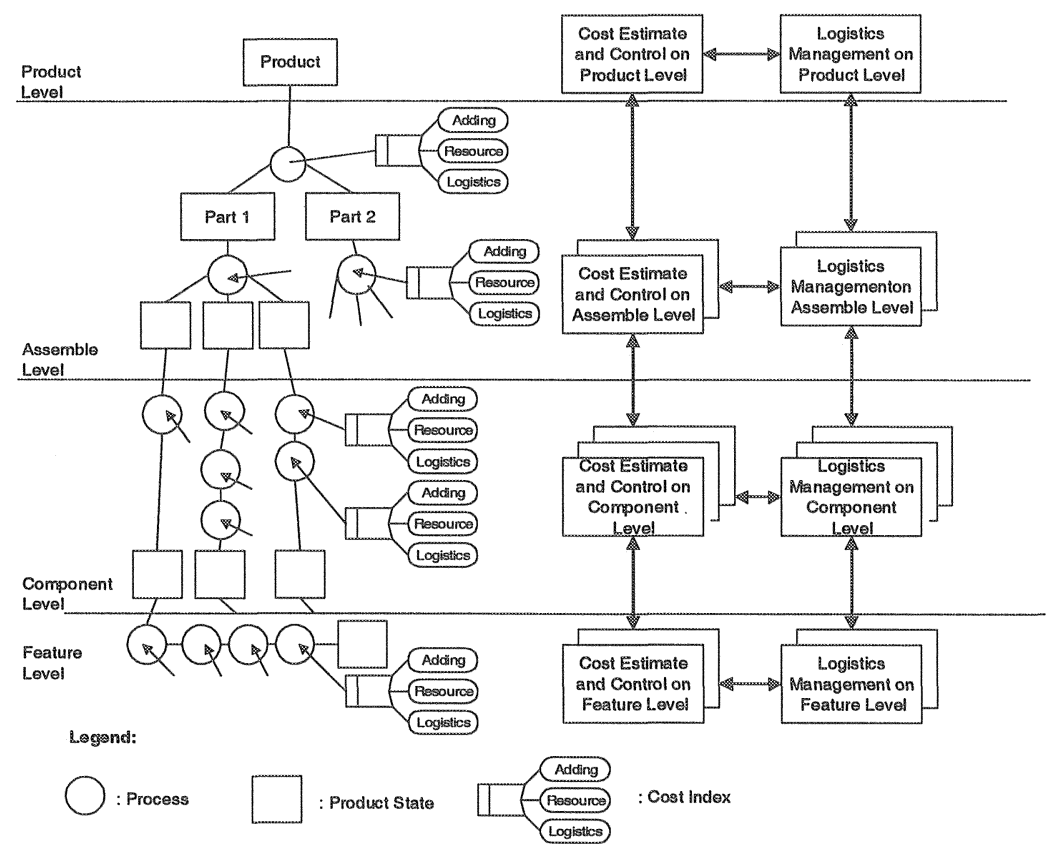

Figure 1 - Cost Index Structure

As shown in Figure 1, a manufacturing process will associated with a cost index. A cost index includes three generic data classes, viz. Adding, Resource and Logistics, which are associated with possible three costs of a manufacturing process as mentioned in previous section. 'Adding' means adding cost to a manufacturing process. It is used to pay for the possible test and reworks due to the difficulty or uncertainty of the process. The difficulty or uncertainty of a process is determined by the design of a product, part, component or feature, e.g. geometry, surface 
finishing or treatment requirements, special tolerance and machining requirements, etc. As discussed in the previously:

- Resource means resource cost or consumption of manufacturing resources as discussed in previous section.

a Logistics means Logistics cost.

Likewise in the Product Production Structure, these cost data classes are written in EXPRESS of STEP so that they can be accessed and retrieved by different computer software systems. The idea to build the Cost Index Structure in a hierarchy on one hand is to keep in line with the overall Product Production Structure. On the other hand, this is also a natural or logic way to estimate, control and record the cost of a product production from feature level on the bottom up to the product level on the top. It is obvious that the cost of a feature, which is added to a component, is a part of the cost of the component. Likewise, the costs of components, which are used to assemble to a part, take the major portion of the part cost, and the product cost will be determined by the costs of the parts which are finally assembled into the product. Hence, the Cost Estimate and Optimal control subsystem estimates the cost of a product production from bottom level up to the top level of the hierarchy as shown in Figure 1. The models, e.g. Cost Estimate and Control models on the Component level, will estimate or search for the cost data (i.e. Adding, Resource, and Logistics) for the generic cost index associated with each manufacturing process by using the cost data from the cost modules on a lower level (e.g. Cost Estimate and Control Models on Feature level), the Costing Knowledge base, and the Logistics Management subsystem. As shown in Figure 1, the Logistics Management subsystem also includes a number of Logistics models which are hierarchically organized from the Feature level on the bottom and up to the Product level on the top respectively.

By the Cost Index Hierarchy as shown in Figure 1, a product production cost can be decomposed from level to level into detailed expenditures or indexes. In fact most of these expenditures on lower levels of the Cost Index Structure can be directly estimated by using the price of a standard component, part, or the cost of a common manufacturing process since to reduce the PD cost the design engineers always try to design the product by using these standard components, parts and common manufacturing processes as many as possible. Also in practice, the costs of quite a few manufacturing processes on a higher level of the Cost Index Structure can be derived by the linear combinations of these standard prices or costs of common processes. Hence, a Costing Knowledge base was developed as an important part of the Cost Estimate and Optimal Control System to structurally record these standard prices and costs of common processes. However, it is difficult to propose a general data structure to build such a costing database for all kinds of manufacturing businesses. The data structure of a Costing Knowledge base for a particular manufacturing business (e.g. sheet metal manufacturing) is some how related to the special manufacturing requirements and practices in that manufacturing.

However, the Cost Index Structure as proposed in Figure 1 is generic, which can be applied for all kinds of manufacturing. To be in line with this cost index hierarchy, the Costing Knowledge base needs to be developed to store the costing 
data in a similar hierarchical structure, i.e. the data tables in the data base are linked and indexed from a table which records the product data information on the top down data tables which record the features data information on the bottom.

\section{COST ESTIMATION AND OPTIMISATION}

With the Cost Index Structure, the cost estimation and optimisation can be described in the following two stages.

Stage 1: Cost estimate: In this stage, the system needs to estimate various costs of the cost indexes associated with processes (See Figure 1) from feature level on the bottom up to the product level on the top. The inputs to the system on this stage include manufacturing processes (including all the possible alternatives) planned by the Manufacturing Process Planning and Optimisation subsystem, manufacturing resources (including all the possible alternatives) and their cost rates and inventory records from the Operations Management subsystem, quotations or prices from all possible suppliers, partners and sub-contractors which are searched and provided by the Logistics Management subsystem, and the costs or prices of these standard components and manufacturing processes from the Costing Knowledge base. With these input data, the Cost Estimate and Optimal Control system will estimate costs or instances for these generic cost indexes associated with the planned processes and their alternatives through using two cost estimate methods, viz. Generative Cost Estimate method and Variant and Knowledge based Cost Estimate method. These two methods will be further described in the following sub-sections. The cost estimate is an incremental or evolutional process, which estimates the cost indexes feature by feature in line with the overall incremental product design concept, and evolutionary production-planning process as implied by the concept of Prototype Based Incremental Product Development (Tu et al. 2000c). It is not developed to estimate various costs of making a product in one-go after a product has been completely designed and planned for production. This incremental way of designing a product, planning the manufacturing processes, scheduling manufacturing resources, controlling inventory, managing logistics and estimating cost feature by feature from the bottom level of a Product Production Structure up to the top level can provide the overall product development system a strong flexibility to cope with the changes and uncertainties in earlier product design stages. Particularly, this incremental way of developing a product and estimating the cost can provide in-time feedbacks or references to support the engineers' decision making on the selection of alternative designs, manufacturing processes, manufacturing resources and alternative suppliers, partners and sub-contractors. The shortages of this incremental method are the lack of global optimisation, and frequent and complicated communication management between these subsystems.

Stage 2: Cost Optimisation: Cost optimisation means a selection among alternative designs, manufacturing processes, manufacturing resources, inventories, suppliers, partners, and subcontractors, or combinations of these alternatives with a lowest cost or total costs. Manufacturing process optimisation, e.g. to find a shortest 
path for a $\mathrm{CNC}$ cutting, and operations optimisation, e.g. an optimal production schedule with a shortest make span, are not the tasks of the cost optimisation. They are optimising tasks of other subsystems, e.g. the manufacturing process planning and optimisation subsystem for sheet metal manufacturing includes a shortest CNC path planning model (Xie et al. 2001a), and the operations management for sheet metal manufacturing include an optimal production scheduling model (Tu 1997a). It is obvious that these optimisations will be carried out before the cost optimisation and they will help the cost reduction. For cost optimisation, we developed an optimal algorithm for the selection of alternative operation routines and supplier chains through using dynamic programming technique (Tu et al. 1997c). In more general, we can use a commercial computer simulation package, e.g. ProModel (by ProModel Corp., Orem, UT), Quest and VNC (by Deneb Inc., Dayton, OH), to develop simulation models to solve cost optimisation problems. If a manufacturing process or a logistic chain consists of random variables or stochastic processes, Markov chains (Zijm 1984) may be used to model the process or the logistic chain and hence a cost optimisation heuristics or algorithm could be developed. This is now under our research.

It should be mentioned that cost optimisation maybe in conflict with other optimisations. An operational routine suggested by the Cost Estimate and Optimal Control subsystem from the lowest cost point of view maybe in conflict with a production schedule suggested by the Operations Management subsystem from the minimum make span point of view. This in fact leads to a problem of decision making with multiple objectives, lowest cost vs. minimum make span. The problem of decision making with multiple objectives can be solved by using rule based or heuristics/algorithm based methods, such as Analytic Hierarchy Process (AHP), Pareto Optimality and Trade-off curves, goal programming (Winston 1994), etc.

\section{Generative Cost Estimate Method}

Generative cost estimate method is a pipeline method. With the Cost Index Structure as shown in Figure 1 and the input data from all the other systems as well as the price and cost data from the costing knowledge base, the Cost Estimate and Optimal Control system can calculate the data or instances of the cost index associated with a process from bottom level up to the top level of the Cost Index Structure. The cost index of punching a hole on a sheet metal (a process on the feature level), for example, can be determined by calculating Adding cost, Resource Cost and Logistics cost. As mentioned before, the adding cost is decided according to the difficulty of the process, i.e. punching a hole on a sheet metal for this example, and it is the sum of the costs of the possible tests and reworks. The resource cost for this example will be calculated by multiplying the standard cost rates of the used manufacturing resources (e.g. punch machine, tool and machine operator) to the used times of these manufacturing resources. The standard cost rates were recorded in the Costing Knowledge base and the used times can be provided by the Manufacturing Process Planning and Optimisation subsystem. The Logistics cost is determined according to whether this process needs to take a material or component into consideration and how and from where this material or component can be provided. To avoid the duplicated cost data in these cost indexes, only the 
first process, which is among a group of sequential processes to be carried out on a blank or a sheet metal, will take the logistics cost of the blank or sheet metal into account. All the other succeeding processes will not take the logistics cost of this blank or sheet metal into account in their cost indexes under the Logistics, or a zero (0) will be assigned to the Logistics. However, if a succeeding process needs to add a new material or component, under the Logistics of its cost index, the logistics cost of this new material or component will be estimated and recorded. As mentioned before, the logistics cost is the sum of the costs of purchased item, transportation (material handling plus inventory cost if the item is in the company), and communication. Normally, a supplier will quote the transportation cost as a part of the quotation for providing an item. Hence the Logistics Management subsystem can provide the costs of purchased item and relevant transportations through inquiring a quotation from suppliers. The communication cost for sourcing items depends on the difficulty of sourcing this item. According to the previous experiences, the management of a company can set up some guidelines or controlled budgets for sourcing various items. These controlled budgets were recorded in the Costing Knowledge base and can be used by the Cost Estimate and Optimal Control subsystem to quote or estimate the communication cost as a part of the logistics cost of getting a required item. If a process is an outsourced process, e.g. subcontracted process, it only has the logistics cost in its cost index and the other two terms will be zero.

When all the cost indexes have fixed data or instances, the production cost of a component, part or product can be calculated by the Cost Estimate and Optimal Control subsystem through adding up all the cost data in the cost indexes associated with these processes which are under and linked (directly or indirectly) to the component, part or product in the Cost Index Structure.

\section{Variant and Knowledge based Cost Estimate Method}

As implied by the discussions made in previous sub-section, the Generative Cost Estimate Method can only be used to estimate the costs of these standard components and processes. For those non-standard or 'new' components and processes which are required by the product design to address particular customer requirements, the variant and knowledge based cost estimate method need to be employed. This method estimates the cost of a component or process through searching and comparing the similarities between the developing product and the products which were made in the past. The Product Production Structures of these past-made products were recorded in a Design/Manufacturing Knowledge base (Tu et al 2000c). The cost indexes are part of the Product Production Structure of a product and hence they must also be record in the Design/Manufacturing Knowledge base. Through searching this Design/Manufacturing Knowledge base, the Cost Estimate and Optimal Control subsystem may find the cost index of a process, which is a part of a past-made product production structure and is similar to the process of the new product. The Cost Estimate and Optimal Control subsystem search for a similar process by following a GT (group technology) coding system (Tu et al. 2000c) and the Product Production Structures are stored and retrieved in the Design/Manufacturing Knowledge base according to the same GT coding 
system. In fact, the similar processes in the Design/Manufacturing Knowledge base have the same process codes. If a process of the new product is a combination of several primary processes, the Cost Estimate and Optimal Control subsystem will search for the cost indexes of the these primary processes and then determine the cost index for this combined process through adding up the cost indexes of these primary processes. A reference number rather than a GT code will be assigned to this combined process which is stored in the Design/Knowledge base as a part of the Product Production Structure. It will not be re-used or searched by the Cost Estimate and Optimal Control subsystem as well as the Manufacturing Process Planning and Optimisation subsystem (Tu et al. 2000c).

\section{Some Considerations on Cost Estimate}

According to the experiences gained from this research and the industrial implementations, by using the two cost estimate methods as discussed in previous subsections, the Cost Estimate and Optimal Control subsystem can estimate cost indexes for most planned manufacturing processes. However, there are still some processes cannot be automatically estimated by the system through using either method. Under this circumstance, the system will show these processes on the screen and ask the product developers to determine the cost indexes for these processes. For a combined process, if the system cannot find the cost indexes for some of its primary processes from the Design/Manufacturing Knowledge base, the system will show these primary processes on the screen, wait for manually costing for these primary processes, and then decide the cost index for the combined process.

Another problem for cost estimate is the cost of a component or outsourced process is within a fuzzy cost domain, i.e. from a lowest to a highest cost. Under this circumstance, a Neural Network based algorithm plus some evaluation rules can be used to inference a most likely cost of the component or the outsourced process. Tu and Jiang (1997a) discussed this algorithm and the evaluation rules.

The third problem in cost estimation and optimisation is a conflict between cost optimisation vs. reliability of suppliers. In practice, the cheapest cost normally implies an un-reliable supplier. The management of a company can solve this problem through choosing proper quality and marketing policies, e.g. only accept quotations from reliable suppliers, partners or sub-contractors.

\section{IMIPLEMENTATION OF THE COST ESTIMATE AND OPTIMAL CONTROL}

The presented Cost Estimate and Optimal Control methods have been implemented in a sheet metal manufacturing company. Through the development of a sheet metal part as shown in Figure 2, we demonstrate the main principles of the cost estimation and optimisation as discussed in the paper. 


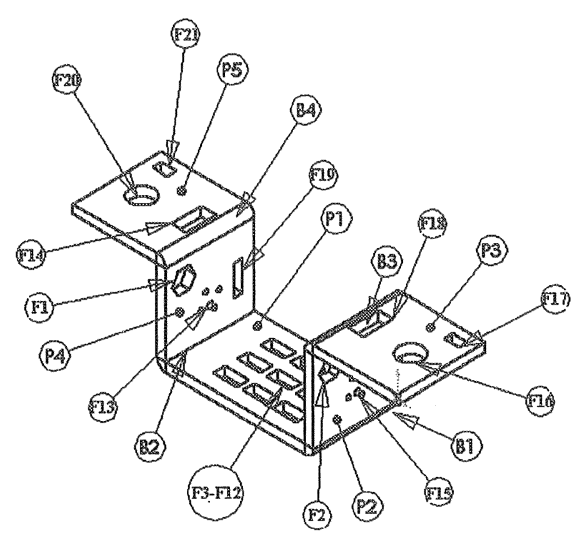

Figure 2 - The case part

\section{Case Scenario and System Description}

As shown in Figure 2, the case part is a sheet metal part which is a part of a sheet metal product. To simplify the discussions here, we only show this sheet metal part instead of the whole sheet metal product.

This sheet metal part can be made by using CNC sheet metal punching machines or a $\mathrm{CNC}$ laser-cutting machine to punch or cut these holes, rectangles, and polygons on a flat sheet metal, and then formed by a bending machine into the final shape. The punching or cutting processes for making these holes, rectangles and polygons are indicated by F1 through F21 as shown in Figure 2. The part will be started as a flat sheet which only includes one face as indicated by P1 in Figure 2. After four bending processes, as indicated by B1 through B4 as shown in Figure 2, the final part will have 5 different faces, i.e. P1 through P5 as shown in Figure 2.

The overall product development system for a sheet metal manufacturing company includes the following subsystems which have the same functions of the sub-systems as discussed in Section 2:

1. Computer aided product definition system: This system was coded by using

Visual C in cooperate with Microsoft Excel. By using the QFD (quality function deployment), the system converts the customer's requirements into the technical attributes of the product (Tu et al. 2002).

2. Computer aided product design system: We use ProEngineer as the computer aided product design system. However, a prototype searching model and an interface model were coded by $\mathrm{C}$ and integrated with the ProEngineer system through its API (application program interface). The prototype searching model is able to search for the prototypes, i.e. products made in the past, which have the most technical attributes to the product to be developed. These prototypes will be further modified into the customer wanted product according to the concept of Prototype Based Incremental Product Development. The interface model is used by the ProEngineer to read and write the Product Production Structure.

3. Manufacturing Process Planning and Optimisation System: This system was coded in $\mathrm{C}++$. To meet the special requirements of sheet metal manufacturing, 
this system includes a nesting model and a CNC tool path-planning model in addition to the process-planning model. The nesting model can be used to automatically plan a cutting layout so that the trim loss can be minimized. The $\mathrm{CNC}$ tool path model was developed to optimally plan the CNC cutting or punching paths so that the total cutting time can be minimized. A screen printout of this system is shown in Figure 3 (Xie et al. 2001a). Figure 3 shows a tool path planning process to be automatically carried out by the system. The parts as shown in Figure 3 have been nested by the system, i.e. properly placed on a given size of sheet so that the material waste or trim-loss is minimized. The part as shown in Figure 2 does not need the nesting optimisation but it needs the tool path planning so that these features could be punched or cut efficiently.

4. Operations Management System: It was coded in $\mathrm{C}++$ and used to schedule shop floor operations and control the inventory through the communication with company's MRP (manufacturing resource planning).

5. Logistics Management System: It was coded in Java. HTML and XML languages as well as Common Gateway Interface (CGI) were used for writing the web pages and manage communications through intra/internets ( $\mathrm{Tu}$ et al. 2000a).

6. Cost Estimate and Optimal Control system: It was code in $\mathrm{C}++$ based on the cost estimate and optimisation methods and algorithms as discussed in Section 4.

All these subsystems can communicate between each other through intra/internets.

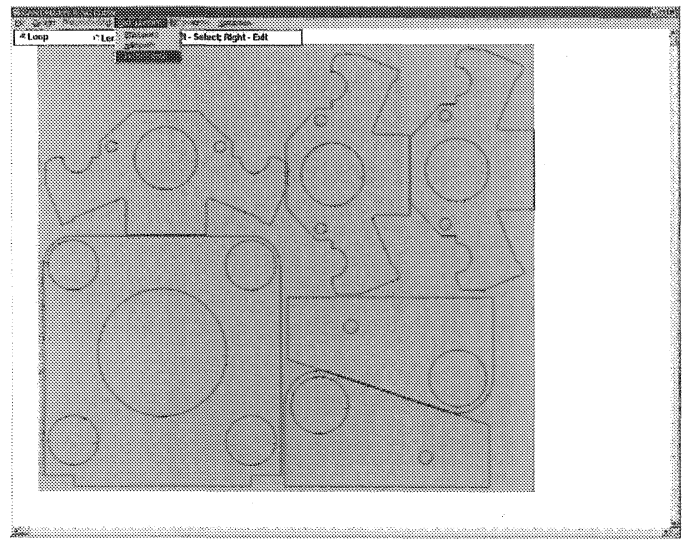

Figure 3 - A screen printout from the Manufacturing Process Planning and Optimisation System for sheet metal manufacturing

\section{Cost Estimate and Optimisation for Making the Case Part}

First, based on the generic Cost Index Structure as shown in Figure 1, the cost index structure for the case part can be drawn and illustrated in Figure 4. As shown 
in Figure 4, the part can be made by sequentially carrying out 24 punching or laser cutting and sheet metal forming processes. Some product states have been inserted into the manufacturing process chain. "P1" means the blank sheet which has one face P1. After folding process B3, the semi-finished part will have two faces, P1 and P3. After the other forming processes, the part will have its final shape which includes 5 faces, P1 through P5. The Manufacturing Process Planning and Optimisation System determine the sequence of the processes as shown in Figure 4.

The first process, i.e. F1 as shown in Figure 4, will consist of the logistics cost in its cost index. The rest of punching or cutting processes will not include logistics cost if they can be carried out in one machine and do not need to be transported to other machines. The first folding process, B3, will include a logistics cost in its cost index. This is the cost for transporting the semi-finished part from the punching or laser cutting machine to a folding machine. The logistics cost of the rest folding processes will be zero if these folding processes can be completed in one folding machine.

In short, by using the methods (including the manual cost estimate) as discussed in Section 4, the cost indexes for all the manufacturing processes can be decided. After these cost indexes have been estimated, the Cost Estimate and Control System would carry out the cost optimisation if it were necessary. As mentioned in Section 4 , the cost estimation is in fact is a selection among alternative designs, manufacturing processes, or operation routines based on cost optimal (or cheapest cost) criterion.

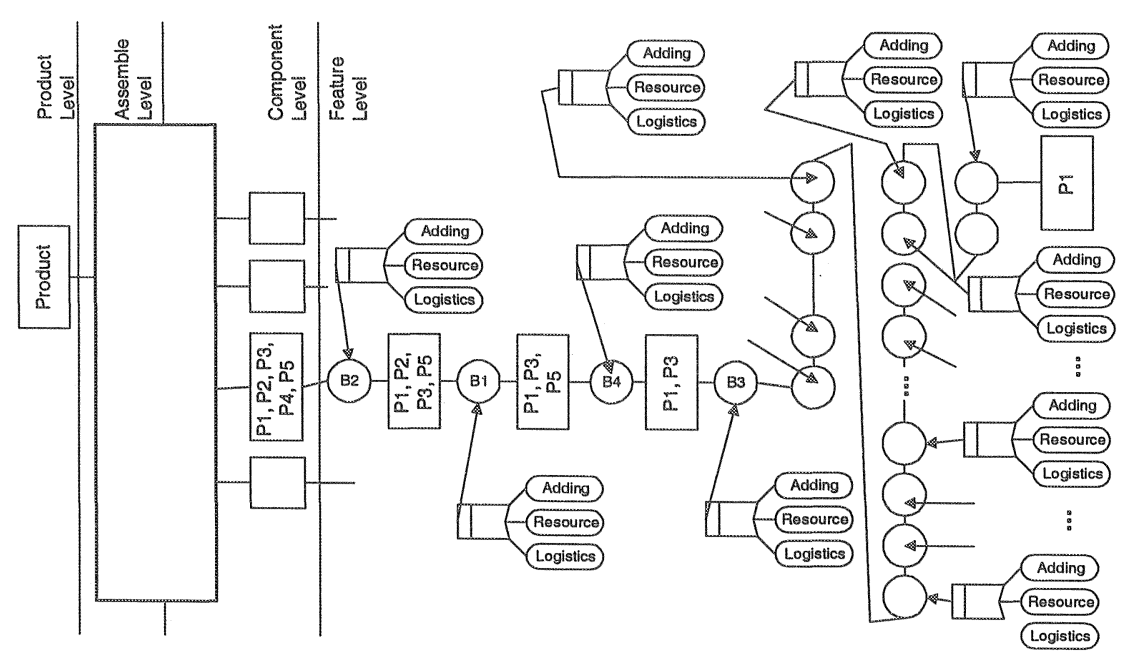

Figure 4-Cosi Index Structure of the case part.

First, let us assume that the 21 machining features (i.e. F1 through F21 as shown in Figure 2) can be carried out in two alternative ways, i.e. 1) to use a CNC punch machine to punch all the 20 features, and 2) to use a laser cutting machine to cut the two polygons ( $F 1$ and $F 2$ ) first and then use a punch machine to punch the rest of holes and rectangles. The first alternative has the advantage of completing all processes in one machine which saves the material handling cost between two 
machines, but it will need a special punch tool to punch these two polygons. The second alternative can have company to avoid having a special punch tool but it implies a material handling cost or logistics cost between the Laser cutting machine and the punch machine, particularly if this laser-cutting machine is an outsourced facility, or a subcontracted process. Under this circumstance, if we ignore other technical factors and only use the cost factor as a reference to make a selection between these two alternatives, this can be done by using the Cost Estimate and Optimal Control system in the following way: 1) The Cost Index Structure as shown in Figure 4 is modified in Figure 5 which includes alternative processes; 2) The Cost Estimate and Optimal Control system estimates costs and decides the cost indexes for all processes; 3) Calculate the total costs for making part by two process alternatives according to the calculation process as described in subsection 4.1. Hence a decision can be made according to these two possible costs.

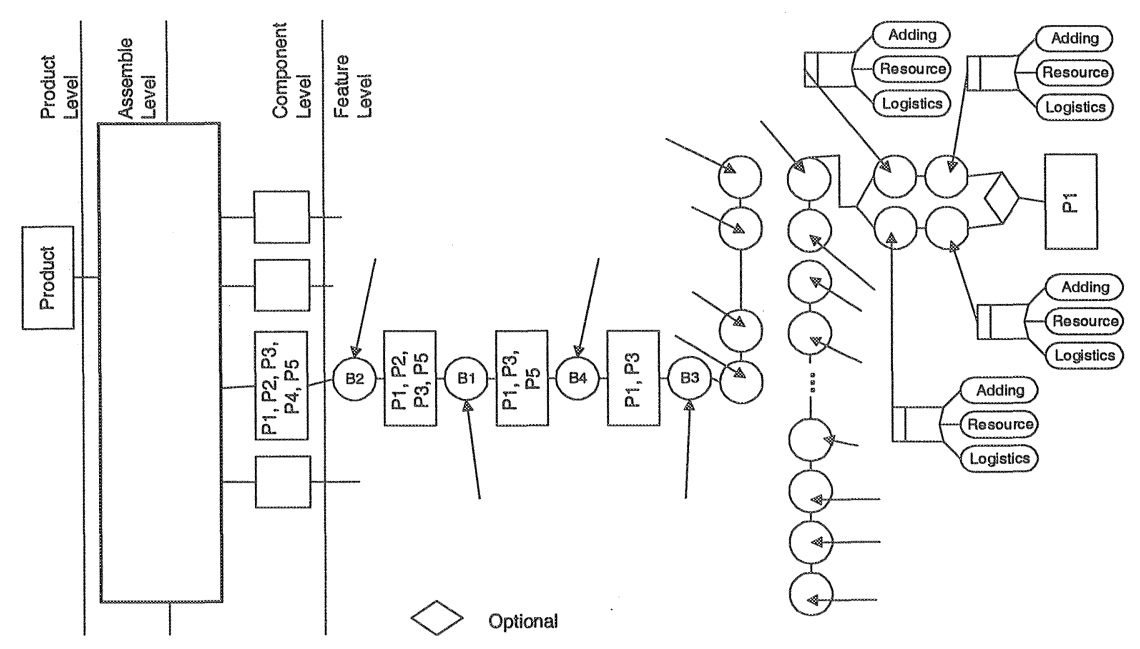

Figure 5 - Cost Index Structure which includes alternative processes

Second, if the case part can be designed in another form, i.e. an alternative design, the Cost Index Structure as shown in Figure 4 will be modified to include alternative designs (or parts) on the Component level). Then these alternative designs will be decomposed into processes on the Feature level respectively. After this modification of the Cost Index Structure, the Cost Estimate and Optimal Control System can estimate the cost indexes for all the processes of the two different designs, and calculate the costs of the two alternative designs, just as the same as it does to make a suggestion to select alternative processes as mentioned in previous paragraph.

Third, if a part can be made by using alternative machines and through alternative operational routines as shown in Figure 6, the dynamic programming cost optimisation model in the Cost Estimate and Optimal Control System can be used to select an optional routine to make the part which has the minimum total cost. Before the Cost Estimate and Optimal Control System can carry out the dynamic programming calculations, it needs to treat these processes on alternative machines 
as alternative processes, record them in the Cost Index Structure, and then estimate the cost indexes for all these alternative processes. Based on the cost indexes of these alternative processes, the system can calculate the cost between a pair of machines, which equals to the machining cost on the next machine plus the material handling cost (or logistics cost) between these two machines. After all the costs between machines in the network as shown in Figure 6 are calculated by the system, the system will use the dynamical programming technique to find a shortest path from the Start point to the Finish point or an operation routine with minimum total cost.

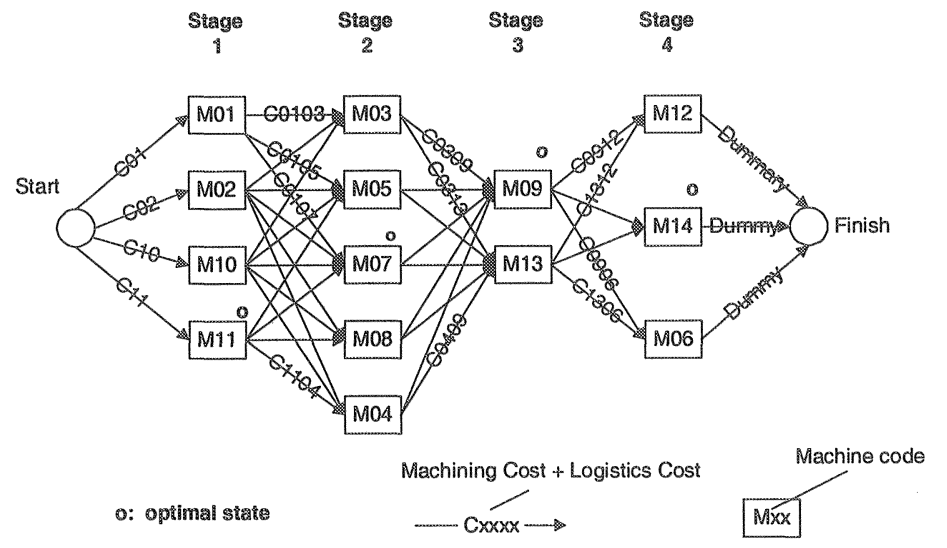

Figure 6 - Dynamic programming networks for making a part through alternative operation routines

This dynamical programming optimisation method can be also applied to select a cheapest logistics chain among a number of alternative chains if we simply think that the machines as shown in Figure 6 are the suppliers, partners or sub-contractors. For a more complicated case in a manufacturing shop floor or a global manufacturing environment, the computer simulation technology, e.g. ProModel and Quest, can be employed for the cost estimation and optimisation. Tu et al. (2000b) reported an application of computer simulation technology to estimate and optimally control the cost of injection mould/tool making in a manufacturing shop floor.

\section{CONCLUSIONS}

The ability to correctly and rapidly estimate and optimally control a product development cost is always a strength which is pursued by manufacturing companies to improve their places in a keen competitive global market. Numerous research efforts have been made to automate the cost estimation and optimisation in a product development cycle although the research and development task is tedious and enormous sometimes. This paper has presented a feasible computer aided solution to automate the cost estimation and optimisation process for control the cost of a product development in a global manufacturing environment. By following the concept of the Prototype Based Incremental Product Development, it is able to 
provide in - time cost estimates and optimisation results to the product developers as important decision-making references in a concurrent engineering approach of the product development. This concurrent approach enables product developers to design the product, plan the processes, schedule operations on the shop floors, manage logistics, and estimate and optimally control the cost in a feature by feature incremental way. This incremental way of developing a product can help the product developers to clarify the product development life cycle data at earlier product development stages and hence avoid reworks which are often occurred in the traditionally sequential approach of a product development. To avoid reworks in a product development cycle, particularly for customized product, will reduce the product development cycle time and cost significantly.

The proposed Cost Index Structure is generic and can be applied to all kinds of products development. It is modelled in EXPRESS of STEP as a part of the Product Production Structure. Hence it can be assessed by all kinds of computer aided engineering or management software systems. It provides links or indexes to associate various costs to product features through the manufacturing processes which are used to create these features. In this way of recording the various costs of a product development, a cost analysis and optimisation in a product development cycle can be easily and automatically done by the Cost Estimate and Optimal Control System as presented in this paper. This cost index structure as a part of the Product Production Structure stored in the Design/Manufacturing Knowledge base can be further used as knowledge and references for future product developments. This is very important for a manufacturing company to record its knowledge and past experiences so that they can be reused to improve the company's practices on product development.

The cost estimate methods and optimisation algorithms are effective. They can be easily understood by and implemented in industry. The Cost Estimate and Optimal Control System as well as other sub-systems of the product development system are running in an Intra/Internet communication environment. This makes these systems or subsystems can run distributed in a computer network. It also improves the system interdependence and reliability, and at same time reduces the complexity of these system design and development. To use the STEP and computer intra/internet communication technology to develop these systems or sub-systems makes these systems very compatible with these commercial computer aided engineering or management software systems, such as ProEngineer, SmartGroup's PDM (product data management) system (Smart Team 2001), etc.

\section{REFERENCES}

[1] ISO 10303-1: 1994, Industrial Automation Systems and Integration - Product Data Representation and Exchange - Part 1: Overview and Fundamental Principles.

[2] ISO 10303-11: 1994(E), Industrial Automation Systems and Integration - Product Data Representation and Exchange - Part 11: The EXPRESS Language Reference Manual.

[3] Smart Team, 2001, SmarTeam Users Guide, Version 4.0, Smart Solutions Ltd, P O Box 5057, Kfar Saba 44150, Israel, www.smarteam.com.

[4] Tu, Y L, and Jiang, Z B, 1997a, "A Neural Network Based Approach for the Quotation of a Product in One-of-a-Kind Production", Proceedings of 1 st International Conference 
on Engineering Design and Automation, March 18-21, 1997, Bangkok, Thailand, pp.307 $-310$.

[5] Tu, Y L, 1997a, "Real-time Scheduling and Control of One-of-a-Kind Production", the International Journal of Production Planning \& Control, Vol. 8, No. 7, 1997, pp. 701 710 .

[6] Tu, Y L, 1997c, "Production Planning and Control in a Virtual OKP Company", the international journal of Computers in Industry, Vol. 34, 1997, pp. 271 - 283.

[7] Tu, Y L, and Xie, S Q, 2000a, "A WWW-based Integrated Product Development Information Management System", Proceedings of IFAC Symposium on Manufacturing, Modelling, Management and Control (MIM 2000), 12-14 July 2000, Patras, Greece, pp. 445 - 450.

[8] Tu, Y L, Xie, S Q, and Fung, R Y K, 2000b, "Rapid Product Development in a Virtual Production Environment", Proceedings of IFAC Symposium on Manufacturing, Modelling, Management and Control (MMM 2000), 12-14 July 2000, Patras, Greece, pp. $63-68$.

[9] Tu, Y L, Chu, X L, and Yang, W Y, 2000c, "Computer Aided Process Planning in Virtual One-of-a-Kind Production", the international journal of Computers in Industry. Vol. 41, 2000, pp. $99-110$.

[10] Tu, Y L, Kam, J J, Fung, R Y K, and Tang, J F, 2002, "Resource Deployment in Earlier Product Development Stages," to appeared on Proceedings of Fourth International Symposium on Tools and Methods of Competitive Engineering, April 22-26, 2002, Wuhan, P R China.

[11] Winston, W L, 1994, Operations Research: Applications and Algorithms, $3^{\text {rd }}$ Edition, Chapter 14, Duxbury Press.

[12] Zijim, H., 1984, "The optimality equations in multichain denumerable state Markov decision processes with the average cost criterion: The unbounded cost case, C. Q. M. Note 22," Centre for Quant. Methods, Philips B.V. Eindhoven, The Netherlands. 